SCIENTIFIC LETTER

\title{
Radial approach to day case intervention in coronary artery lesions (RADICAL): a single centre safety and feasibility study
}

\author{
S Kumar, R Anantharaman, P Das, J Hobbs, C Densem, J Ansell, D H Roberts
}

Heart 2004;90:1340-1341. doi: 10.1136/hrt.2003.021246

$\mathrm{T}$ ransradial arterial access for percutaneous coronary intervention (PCI) is increasing in popularity. Its advantages are a significant reduction of vascular complications at the access site and immediate mobilisation. ${ }^{1}$ Same day discharge following transradial PCI is therefore possible and we report our initial experience with a consecutive group of 150 patients treated in a regional cardiac centre over a period of 21 months.

\section{METHODS}

Patients listed for elective PCI were initially selected for potential day case PCI on clinical (Canadian Cardiovascular Society class I-III) and angiographic criteria (complex lesions in degenerated saphenous vein grafts at risk of producing distal embolisation, bifurcation lesions involving a significant side branch of more than $2 \mathrm{~mm}$ diameter, and unprotected left main stem stenosis were excluded) by the consultant cardiologist (DHR). During further assessment in the preadmission stage patients were excluded on clinical (excessive co-morbidity) and social (no carer available at home on the night of the procedure) criteria. A non-ischaemic Allen's test was required, ensuring adequate ulnar collateral supply. All patients were prescribed aspirin $300 \mathrm{mg}$ and clopidogrel $300 \mathrm{mg}$ in the pre-admission clinic and were instructed to take them on the evening before the procedure.

PCI (2-3 cases) was performed in a morning session by one consultant and a supervised specialist registrar as first or second operator. Following successful radial puncture, intraarterial drugs were administered to prevent radial spasm (200 $\mu \mathrm{g}$ of glyceryl trinitrate and $2.5 \mathrm{mg}$ verapamil) followed by the insertion of a Cook 6 French, $23 \mathrm{~cm}$ hydrophilic sheath. Intravenous heparin (70 units $/ \mathrm{kg}$ ) was routinely administered. Guide catheter selection was similar to the femoral approach with a half French size reduction in the left coronary catheter in most cases. Direct stenting was attempted in most cases of non-occlusive disease. An abciximab bolus $(0.25 \mathrm{mg} / \mathrm{kg})$ was administered at the discretion of the operator.

Following successful PCI the arterial sheath was immediately removed and mechanical occlusive pressure with Radistop (Radimedical) applied. All patients were ambulated almost immediately after PCI, provided no sedation was used, and returned to the adjacent day case ward for observation. A routine ECG and a platelet count were performed four hours after the procedure (for those who had received abciximab). They were discharged within 30 minutes of Radistop removal. All patients were followed up by the referring cardiologist at six weeks. Procedural day case success was defined at this point as successful PCI of all target lesions with less than $25 \%$ residual stenosis with TIMI (thrombolysis in myocardial infarction) 3 flow, no complications, and same day discharge. Patients were given written hospital support details before discharge and were routinely contacted by telephone the following day. A patient satisfaction questionnaire was also used to evaluate the day case procedure.

\section{RESULTS}

The clinical profile of patients selected for day case intervention is shown in table 1 . Patients initially selected had single vessel non-occlusive disease but the case mix rapidly broadened. With this case mix, day case discharge was successful in $80 \%$ of patients. Day case failures fell into three groups. The first group involved difficulties inherent in the radial access such as failed cannulation $(\mathrm{n}=4)$, inability to access the ascending aorta $(\mathrm{n}=5)$, or poor guide catheter support $(n=4)$. The second group were coronary artery dissections $(n=4)$, all of them successfully treated by stenting and abciximab infusion, or failure to reopen chronic total occlusions $(n=7)$. Of these seven patients all were discharged the same day with referral for coronary artery bypass grafting in four patients and medical treatment in three patients. One patient returned to the catheter laboratory with chest pain and ST elevation and was found to have acute stent thrombosis. He was successfully treated with percutaneous transluminal coronary angioplasty (PTCA) and abciximab infusion and was discharged the following day. A third category of patients were kept overnight at the discretion of the operator for a variety of reasons including: an extravassated intravenous cannula $(\mathrm{n}=1)$; transient

Table 1 Profile of patients in the radical study

\begin{tabular}{ll}
\hline Mean age & 54.6 years \\
Females & $\mathrm{n}=50(33 \%)$ \\
Patients with diabetes & $\mathrm{n}=27(18 \%)$ \\
Single vessel & $\mathrm{n}=112(80.6 \%)$ \\
Two vessel & $\mathrm{n}=25(18 \%)$ \\
Three vessel & $\mathrm{n}=2(1.4 \%)$ \\
Lesion type & \\
A & $28.8 \%$ \\
B1 & $34.6 \%$ \\
B2 & $8.8 \%$ \\
C & $27.5 \%$ \\
Vessel diameter & $2.25 \mathrm{~mm}-4 \mathrm{~mm}$ \\
Mean number of stents per patient & 2.1 \\
Mean length of stent per patient & $34 \mathrm{~mm}$ \\
Mean fluoroscopy time & $13.6 \mathrm{minutes}$ \\
Mean procedure time & $58.8 \mathrm{minutes}$ \\
Mean contrast volume & $240 \mathrm{ml}$ \\
Direct stent & $63 \%$ \\
Abciximab use & $75 \%$ \\
Vessel treated & \\
LAD & $\mathrm{n}=59$ \\
Diagonal & $\mathrm{n}=4$ \\
Circumflex & $\mathrm{n}=27$ \\
Marginal & $\mathrm{n}=11$ \\
RCA & $\mathrm{n}=64$ \\
Vein graft & $\mathrm{n}=2$
\end{tabular}

$L A D$, left anterior descending artery; RCA, right coronary artery 
ischaemic attack $(\mathrm{n}=1)$; severe vasovagal reaction $(\mathrm{n}=1)$; need to give abciximab infusion $(\mathrm{n}=3)$. These patients had progression of their coronary artery disease since diagnostic angiography; they were given abciximab infusion and kept overnight after successful treatment with complex bifurcation stenting techniques. During the 30 day follow up three patients had recurrent angina but there were no representations within this period; one patient died on day 3 from an unrelated cause (30 day major adverse coronary events (MACE) 0.67\%). There were three re-presentations with chest pain caused by in-stent restenosis over the 21 month follow up period. Two were successfully treated with PCI as a further day case radial procedure. None of the patients had access site complications or needed medical assistance for any other reason post-discharge.

\section{DISCUSSION}

Vascular complications following a successful PCI are a major factor limiting early mobilisation and same day discharge. While the use of low dose heparin, smaller sheath size, and arterial closure devices aid earlier mobilisation after transfemoral approach, the transradial approach remains the most predictable method of avoiding vascular complications. Most studies on day case transfemoral approach have been highly selective in their inclusion criteria, thus excluding many of the patients awaiting PCI. ${ }^{2}$ Day case PCI through the radial approach has been evaluated in a pilot study (OUTCLAS) with $40 \%$ stent usage and $66 \%$ same day discharge. ${ }^{3}$ Our same day discharge is higher but still conservative as it includes both our learning curve and failed cases of chronic total occlusions. The case mix includes multiple coronary lesions, multivessel disease, long lesions, and lesions in small vessels. Stents were used in all but two cases, with direct stenting in $63 \%$ of cases. We were able to complete three such cases in the morning session. We have found that day case PCI is popular with patients and nurses. Our subjective patient survey showed that $73 \%$ felt that transradial PCI was a more acceptable procedure than their previous transfemoral day case coronary angiogram. Ten per cent felt that the transfemoral approach was better. The latter subset had more radial site discomfort than the first group. These findings were independent of the use of analgesia.

The close proximity of the day case unit allowed more consultant input into the package of care. We felt that we were able to provide more patient education in the day case unit where the nurse to patient ratio is $3: 8$, compared to $3: 16$ in the general cardiology ward where patients who needed overnight stay following femoral PCI are admitted. One of the disadvantages of a day case procedure is the inability to measure a routine troponin concentration before discharge, but we had one MACE in the first 30 days. All patients were pre-treated with aspirin and clopidogrel using guidelines similar to the recently presented CREDO trial. ${ }^{4}$ An important and unresolved issue from this study remains the appropriate use of abciximab. ${ }^{5}$ A weight adjusted bolus of abciximab was given to patients at higher risk of stent thrombosis and did not prolong radial compression time (4 hours and 22 minutes in the abciximab group and 4 hours and 24 minutes in the non-abciximab group) or prevent same day discharge. Abciximab infusion with overnight stay was reserved for complications from PCI. The single case of stent thrombosis in this study occurred in a patient who did not receive an abciximab bolus after a single stent implantation, but who in retrospect had been found to be inadequately pre-treated with clopidogrel. Many of our patients travel up to 60 miles each way for the procedure, making the use of a strict protocol based pre-assessment mandatory.

Although transradial PCI has a learning curve we believe this technique is the most suitable approach for day case PCI. It enables efficient management of the interventional workload through day case beds and can increase throughput without major change in infrastructure. Apart from the obvious economic incentives from outpatient PCI, there is a good perception of the procedure by the patients. The RADICAL study firmly establishes the safety and feasibility of this approach in elective interventional practice in the UK.

\section{Authors' affiliations \\ S Kumar, R Anantharaman, P Das, J Hobbs, C Densem, J Ansell, \\ D H Roberts, Blackpool Victoria Hospital, Blackpool, Lancashire, UK}

Correspondence to: Dr Somnath Kumar, Department of Cardiology, Blackpool Victoria Hospital, Whinney Heys Road, Blackpool, Lancashire FY3 8NZ, UK; somnathkumar@hotmail.com

Accepted 18 December 2003

\section{REFERENCES}

1 Kiemeneij F, Laarman GJ, Odekerken D, et al. A randomised comparison of percutaneous transluminal coronary angioplasty by the radial, brachial and femoral approaches:the access study. J Am Coll Cardiol 1997;29:1269-75.

2 Banning AP, Ormerod OJ, Channon K, et al. Same day discharge following elective percutaneous coronary intervention in patients with stable angina. Heart 2003;89:665.

3 Slagboom T, Kiemeneii F, Laarman GJ, et al. Actual outpatient PTCA: results of the OUTCLAS pilot study. Catheter Cardiovasc Interv 2001;53:204-8.

4 Steinhubl SR, Berger PB, Mann JT, et al. Early and sustained dual oral antiplatelet therapy following percutaneous coronary intervention: a randomised controlled trial. JAMA 2002;288:2411-20.

5 Kastrati Adnan, et al. Abciximab not needed in elective $\mathrm{PCl}$ if high loading dose of clopidogrel used, Abstract presentation, American College of Cardiology, 2003 scientific session. 